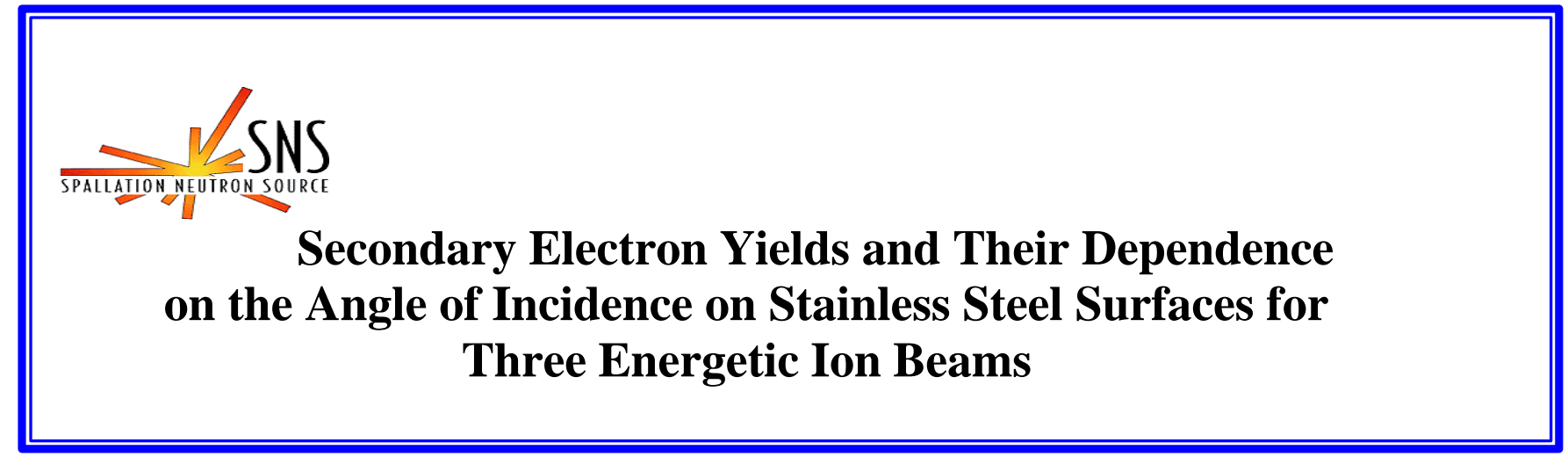

BNL/SNS TECHNICAL NOTE

NO. 064

P. Thieberger, A. L. Hanson, D. B. Steski, V. Zajic,

S. Y. Zhang and H. Ludewig

August 18, 1999

ALTERNATING GRADIENT SYNCHROTRON DEPARTMENT

BROOKHAVEN NATIONAL LABORATORY

UPTON, NEW YORK 11973 


\title{
Secondary Electron Yields and Their Dependence on the Angle of Incidence on Stainless Steel Surfaces for Three Energetic Ion Beams
}

\author{
P. Thieberger, A. L. Hanson, D. B. Steski, V. Zajic, S. Y. Zhang and H. Ludewig \\ Brookhaven National Laboratory, Upton NY, USA
}

\begin{abstract}
Secondary electron yields were investigated for $28 \mathrm{MeV}$ protons, $126 \mathrm{MeV}$ oxygen ions and $182 \mathrm{MeV}$ gold ions incident on 304 stainless steel surfaces. The dependence on the incidence angle was studied in detail, and a system was developed which allows accurate measurements to be performed over a wide angular range extending to nearly grazing collisions. Electron yield estimates of interest for future accelerator applications are developed for $1 \mathrm{GeV}$ protons, and the possible mitigation of deleterious effects by using serrated rather than flat surfaces is analyzed.
\end{abstract}

\section{Introduction}

There is considerable experimental information available for the yield of secondary electrons ejected following the impact of a variety of ions on different solid surfaces and also at the exit surfaces for ions penetrating thin targets, and the field has been recently reviewed by several authors [1-4]. There are relatively few measurements for energies larger than $1 \mathrm{MeV} / \mathrm{amu}$, and of these, most are performed at normal incidence. Near grazing collisions are of particular importance for the practical applications described below. They are also of interest for the understanding of the underlying phenomena, since the yields (which become very large) are known, at lower energies, to deviate markedly from predictions based on semi-empirical theories.

The present experiments were motivated by the need to evaluate (and eventually avoid or mitigate) deleterious effects of secondary electrons on ion accelerator performance. In particular, e-p instabilities could occur in the Spallation Neutron Source (SNS) now being built, if large numbers of electrons are generated through grazing collisions of halo protons with the surfaces of the collimators which are an essential part of the SNS ring design [5]. Also, loading effects noticed at the AGS Booster inflector when 1 $\mathrm{MeV} / \mathrm{amu}$ gold beams are injected are clearly due to secondary electrons. In fact one of us (S.Y. Zhang) recently performed measurements of this effect [6] and arrived at preliminary yield estimates for grazing collisions of $1 \mathrm{MeV} / \mathrm{amu}$ gold ions with a stainless steel surface. There are very few other measurements of electron yields for stainless steel (SS) in the literature, and only at much lower energies (see e.g. [7]). This material was chosen for the present work because of its importance for the accelerator applications and also because it allows one to largely avoid the issue of surface oxide layers drastically influencing the results under realistic vacuum conditions.

For ion energies of interest here (> $100 \mathrm{KeV} / \mathrm{amu}$ ) almost all of the electrons emitted into the vacuum following the ion entrance into (or the exit from) a solid surface come from within the solid. The production of these electrons can be described as a three-step process [2]. First the incoming ion transfers energy to electrons in the solid at a rate given by $(\mathrm{dE} / \mathrm{dx})_{\mathrm{e}}$ the electronic stopping power, which for the ions of interest here is by far the largest part of the total stopping power (see Table 3 below). Second, the electrons scatter and cascade, multiplying and diffusing through the solid. Finally, a small fraction of them, mostly originating from an escape zone or surface layer, which e.g. for carbon is typically $\sim 30 \AA$ thick [8], manage to penetrate the surface potential barrier escaping into the vacuum. Since this escape zone is so thin, the electronic stopping power relevant for the escaping electrons can be evaluated at the incident ion energy.

To the extent that the above picture is valid, one can expect $[9,10]$ the thick target backward yield $\gamma_{\mathrm{B}}$ (i.e. mean number of electrons emitted backwards per incident ion) to be: 
$\gamma_{\mathrm{B}}=\Lambda_{\mathrm{M}} \beta_{\mathrm{S}}\left(\frac{\mathrm{dE}}{\mathrm{dx}}\right)_{\mathrm{e}} \cos ^{-1}(\theta)$

Where $\Lambda_{\mathrm{M}}$ is a constant for a given material, and $\theta$ is the angle of incidence with respect to a line perpendicular to the surface. $\beta_{S}=1-\beta_{\delta}$ is the partition factor for "soft" collisions which describes the fraction of the projectile energy lost directly to low energy electrons, and $\beta_{\delta}$ is the fraction lost in close collisions leading to the more energetic $\delta$-electrons. These $\delta$-electrons are mostly forward directed and are therefore thought to make a negligible contribution to the backward yield $\gamma_{\mathrm{B}}$. Sternglass [11] had assumed an equipartition between both types of collisions for fast projectiles, i.e. $\beta_{S}=\beta_{\delta}=0.5$ which would lead to a Meckbach factor [12] $\gamma_{\mathrm{F}} / \gamma_{\mathrm{B}}=2$ where $\gamma_{\mathrm{F}}$ is the forward yield. Experimental values of this ratio are usually smaller, e.g., $\sim 1.2$ for protons of 0.02 to $9.5 \mathrm{MeV}$ on carbon targets [10].

Equation [1], especially for $\theta=0^{\circ}$, holds fairly well for protons over a wide energy ranges extending e.g. from 0.02 to $7.5 \mathrm{MeV}$ for carbon targets [13] and from 15 to $68 \mathrm{MeV}$ for a $\mathrm{Al}_{2} \mathrm{O}_{3}$ target [14]. Deviations encountered for heavier ions both in yield at normal incidence, and in angular dependence can be parametrized as follows $[15,2]$

$\gamma_{\mathrm{B}}(\theta)=\gamma_{\mathrm{B}}\left(0^{\circ}\right) \cos ^{-\mathrm{f}}(\theta)=\mathrm{C}_{\mathrm{B}} \Lambda_{\mathrm{B}}^{\mathrm{Z}=1}\left(\frac{\mathrm{dE}}{\mathrm{dx}}\right)_{e} \cos ^{-\mathrm{f}}(\theta)$

where, by definition, $C_{B}=1$ for protons, and $\Lambda_{B}{ }^{Z=1}$ is the ratio $\gamma_{B}{ }^{Z=1}\left(0^{\circ}\right) /(d E / d x)_{e}$ for protons at normal incidence. As mentioned above, for a given target material, this ratio has been found to be fairly constant over several orders of magnitude in energy. $\Lambda_{\mathrm{B}}^{\mathrm{Z}=1}$ is therefore a parameter characteristic of each material. The ratio $\gamma_{\mathrm{B}}\left(0^{\circ}\right) /(\mathrm{dE} / \mathrm{dx})_{\mathrm{e}}$ for other ions is usually smaller than for protons and such "deficits" are reflected in values of $C_{B}<1$. Deviations from the simple description summarized in eq. 1 will thus be quantified as values of the parameters $C_{B} \neq 1$ and $f \neq 1$

Several possible causes have been mentioned in the literature for values of $C_{B}<1$, i.e. for less efficient energy conversion into back-scattered electrons for heavy ions as compared to protons $[2,10]$. One explanation is based on the fact that, while traversing the thin escape zone, the ion usually is far from the equilibrium charge state, and therefore the effective value of $(\mathrm{dE} / \mathrm{dx})_{\mathrm{e}}$ will in general be different (usually smaller) than the bulk value. Such near-surface nonequilibrium stooping-power effects have in fact been observed with ions of equal velocities and different charge states [16].

Describing the deviation from the $1 / \cos (\theta)$ behavior by a factor $\mathrm{f} \neq 1$ in eq. 2 is a purely empirical approach [2] which so far has proven to fit data fairly well for $0^{\circ}<\theta<80^{\circ}$ (see e.g. [17]). For angles close to $90^{\circ}$ obviously data must (and do) deviate even from this behavior [17]. In general, deviations from the 1/ $\cos (\theta)$ law can be expected for increasing angles if the mean value $(\mathrm{dE} / \mathrm{dx})_{\mathrm{e}}$ changes as increasingly long segments of the track come close enough to the surface for electrons to escape. ( $\mathrm{dE} / \mathrm{dx})_{\mathrm{e}}$ may be changing significantly either by the gradual charge equilibration mentioned above or as a consequence of energy loss of the ion.

Other possible reasons [2] for deviations from the $\cos ^{-1}(\theta)$ dependence have to do with the fact that the ion trajectories will deviate from a straight lines due to multiple scattering. At grazing angles some fraction of the ions will scatter from the target, but this effect is more significant at lower ion energies. There is also the possibility that, in the cascade leading to the observed electrons, memory may not be totally lost of the initial angular correlations of scattered electrons with respect to the direction of the incoming ion.

For the present experiments ions were selected with charge states close to their equilibrium value for solid strippers. The interpretation of the data should thus be somewhat simplified by avoiding those of the abovementioned complications which are related to rapidly changing charge states. The ions utilized were protons and fully stripped oxygen at $28 \mathrm{MeV}$ and $126 \mathrm{MeV}$ respectively (which, at the Brookhaven Tandem, are close to the maximum achievable energies for these ions), and $182 \mathrm{MeV}$ gold with 31 electrons removed. This $182 \mathrm{MeV}$ gold beam has the same energy and charge state as the one utilized to inject the AGS Booster for the Brookhaven relativistic heavy ion program and for the Relativistic Heavy Ion Collider (RHIC) [18]. 
The aim of the present work was to perform accurate electron yield and angular dependence measurements starting at normal incidence and approaching grazing incidence as closely as possible, and to determine to what extent the use of serrated surfaces could suppress the large yields characteristic of grazing collisions.

We describe the experimental arrangement in section II and present the data and the result of least square fits in section III. Discussion of the flat plate data and comparisons with other results are presented in section IV, the serrated plate results and prospects for higher energies are analyzed in section V, and some conclusions are reached in section VI.

\section{Experimental Arrangement}

Figure 1a is a schematic (not to scale) top view of the experimental arrangement. The ion beam comes in from the left through two sets of collimating slits S1 and S2 and a fixed cleanup aperture C. It either hits the $305 \mathrm{~mm}$ long target plate $\mathrm{T}$, or it continues through a $6.35 \mathrm{~mm}$ high center slot (see fig. 1b) to be measured in the Faraday cup FC2. An anode plate A parallel to $\mathrm{T}$ is mounted at $25.4 \mathrm{~mm}$ from $\mathrm{T}$ on insulating posts. This anode plate has three $9.5 \mathrm{~mm}$ high slots to allow the beam to either go trough the slot in $\mathrm{T}$ or to hit the surface of $\mathrm{T}$ above or below this slot depending on the vertical position of the plate assembly. The anode $\mathrm{A}$ is also provided with a box $\mathrm{B}$ designed to capture most electrons that may escape through the slots in A, while still allowing the ion beam to get in for all angles $2>$ $55^{\circ}$ through slots in the front of the box, and for angles around $50^{\circ}, 40^{\circ}, 20^{\circ}$ and $0^{\circ}$ through the apertures in the side of the box as schematically indicated in fig. 1a. These apertures, which are normally provided with covers, are selectively uncovered to obtain data at the desired angles.

The plate assembly is mounted on the center post of a vacuum chamber by means of insulating adjustable alignment screws (not shown). The vertical position of this post can be remotely controlled as well as the angle 2 which is measured by means of a digital rotary angle encoder. The position of the target plate $\mathrm{T}$ is accurately adjusted so that the axis of rotation be in the plane of the target and the top of the plate be level.
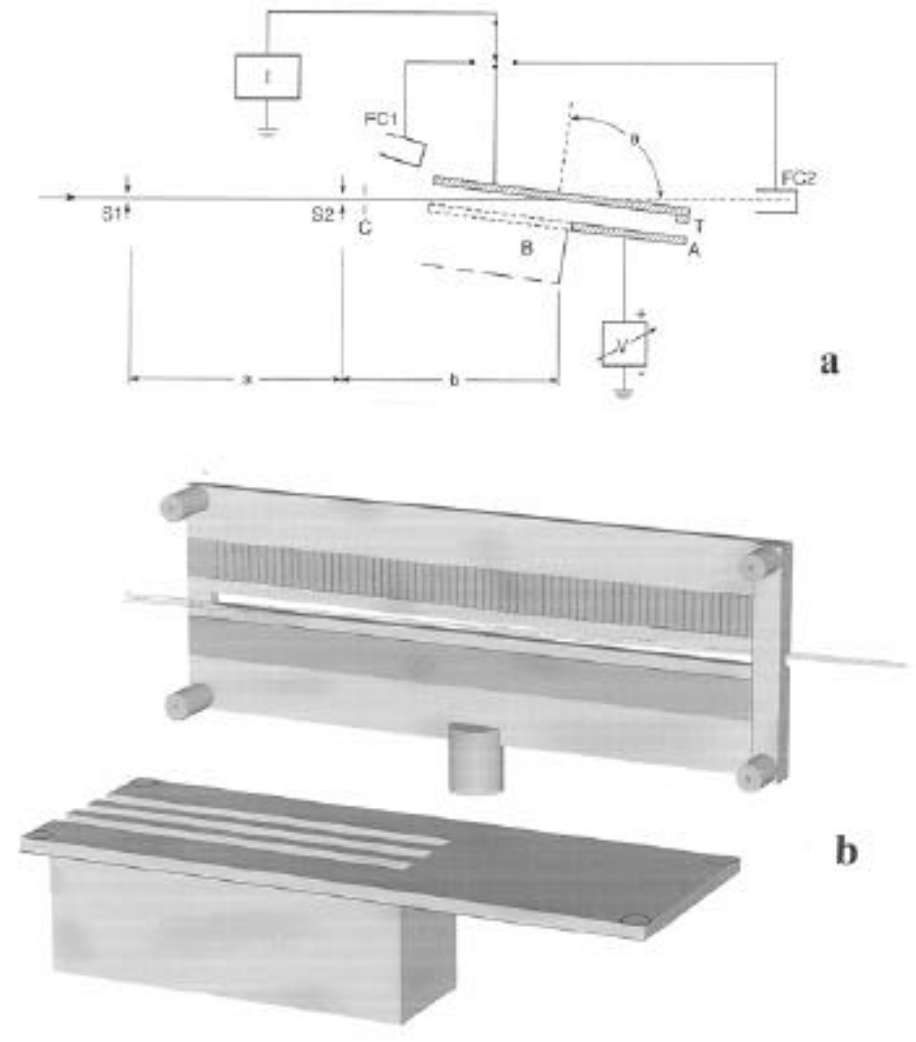

Figure 1 a) Schematic diagram of the experimental arrangement. The horizontal scale is distorted. The distance a between the slits S1 and S2 is $2031 \mathrm{~mm}$, the distance b between S2 and the center of plate $\mathrm{T}$ is $622 \mathrm{~mm}$ and the length of the plates is $305 \mathrm{~mm}$. See text and Table 1 for details. b) Perspective representation of the plate assembly. Plate $\mathrm{T}$ is schematically shown as mounted on a large cylindrical insulator, the axis of which is the axis of rotation. In reality this plate was mounted on a stage with insulating adjustment screws for the purpose of alignment. Plate A, shown at the bottom removed from the assembly, was supported by the four small insulating posts shown protruding from plate $\mathrm{T}$.

The target plate shown in fig. $1 \mathrm{~b}$ has two inserts, one of which is flat and the other one is serrated. The idea was to compare the yields form both types of surfaces in a single measurement. This was done, but the flat-plate data presented in the next section was obtained with a similar but entirely flat plate without inserts. The reason for this is that the 
alignment and flatness of the inserts were not good enough to allow accurate measurements for angles $2>$ $89.5^{\circ}$. Thus, for the final data, the plate with the inserts was only used to obtain yields from the serrated surface, for which the last few tenths of a degree are of less interest.

A Faraday cup FC1 can be introduced between the cleanup aperture and the plate assembly. For most measurements the anode plate was positively biased (200 Volts), and the electron current superimposed with the beam current was measured in an electrometer which was also used to measure currents in the two Faraday cups. Only for the proton beams at angles $2<70^{\circ}$ was it necessary instead to bias the target $\mathrm{T}$ negatively (-200 Volts), and to measure the electron current on the anode A. For those points the electron yield becomes much smaller than 1, and can no longer be accurately measured when added to the much larger beam current.

The physical dimensions most relevant for the flat plate measurements are defined and listed in Table 1. The last two quantities in Table 1 were computed using the above given slit openings $h_{s 1}$ and $h_{s 2}$ and the distances $a$ and $b$ (see fig. 1a). The slits are adjustable and wider openings were used for a number of preliminary measurements. The values shown here correspond to the data presented in the next section for the flat plate. For the less critical serrated plate measurements, slit settings were utilized which were about twice as large as indicated in Table 1 . The corresponding full horizontal beam size and the horizontal angular Table 1. Physical dimensions for the flat plate measurements. beam spread for the

\begin{tabular}{|l|c||}
\hline Distance a between slits S1 and S2 & $2031 \mathrm{~mm}$ \\
\hline Distance b between S2 and the center of rotation & $622 \mathrm{~mm}$ \\
\hline Total horizontal opening $\mathrm{h}_{\mathrm{s} 1}$ of slit S1 & $1.0 \mathrm{~mm}$ \\
\hline Total horizontal opening $\mathrm{h}_{\mathrm{s} 2}$ of slit S2 & $0.88 \mathrm{~mm}$ \\
\hline Total length L of the plate T & $305 \mathrm{~mm}$ \\
\hline Length L' of the open slot in plate T & $279 \mathrm{~mm}$ \\
\hline Height of the slot in plate T & $6.35 \mathrm{~mm}$ \\
\hline Flatness tolerance for plate T & $\pm 0.025 \mathrm{~mm}$ \\
\hline Full horizontal beam size at center of plates & $1.46 \mathrm{~mm}$ \\
\hline Horizontal angular beam spread & $\pm 0.027^{\circ}\left( \pm 1.6^{\prime}\right)$ \\
\hline
\end{tabular}
serrated plate data are respectively $2.8 \mathrm{~mm}$ and $\pm 0.05^{\circ}$.

One problem encountered when attempting precise electron yield measurements closer and closer to grazing incidence angles is that, in spite of tight collimation and long plates, beyond a certain angle the beam spot size on the target will exceed the target length. For the present case the angle for which the beam spreads out over just the entire length of the plate is $\theta=89.73^{\circ}$ as is easy to compute from the values given in Table 1. Unavoidable misalignments may actually cause some of the beam to start missing the target surface at slightly smaller angles. Thus, to get closer to $90^{\circ}$ one must devise a system that compensates for such losses or that allows appropriate corrections to be made.

When the beam goes through the slot in the target plate $\mathrm{T}$ (see fig. 1a) there should normally be no current measured from this plate until, approaching angles close $90^{\circ}$, some of the beam will no longer go through. Portions of the beam are then intercepted by both the $12.7 \mathrm{~mm}$ wide tab connecting the upper and lower portions of the plate at the left, and by the $12.7 \mathrm{~mm}$ wide vertical bar mounted in contact with the plate surface and covering the end of the slot at the right. The Faraday cup FC2 current is recorded as well as the plate signal. The plate signal is then subtracted from the signal obtained for the same angle when the plate assembly is moved to the up or down positions where the target plate intercepts the full beam. This difference is then a measure of the signal one would obtain solely from the fraction of the beam hitting a length of plate equal to the open length of the slot. The Faraday cup FC2 current previously recorded corresponds precisely to that fraction of the beam, and therefore the yield can be determined. Under these circumstances the useful part of the beam results from further collimation and therefore the effective angular beam spread will in general be even smaller than indicated in Table 1.

The above mentioned subtraction of plate signals is in fact carried out for all angles even though the subtracted signal becomes negligible for angles $\theta<89.5^{\circ}$. Small spurious signals, such as those due to residual gas ionization or to electrons originating from the slits, would thus be cancelled. 
For the configuration shown in fig. 1a, i.e. positive bias applied to the anode A and current measured from the target $\mathrm{T}$, the measured yield at a given angle 2 is calculated as follows:

$\gamma_{\mathrm{B}}(2)=\mathrm{q} \times\left(\frac{\mathrm{I}_{\mathrm{t}}-\mathrm{I}_{\mathrm{t} 0}}{\mathrm{I}_{2}}-1\right)$

Where $\mathrm{q}$ is the charge state, $\mathrm{I}_{\mathrm{t}}$ is the target current measured in the up or down position, $\mathrm{I}_{\mathrm{t} 0}$ the target current measured in the center position with beam going through the slot, this beam being measured as $I_{2}$ in the Faraday cup FC2.

When the yield is much smaller than 1, the signal from the electron current is swamped by the beam current and the accuracy of the measurement then requires the target to be biased negatively and the anode currents to be measured instead of the target currents. Calling the anode currents $I_{a}$ and $I_{a 0}$ for the up or down and for the center positions respectively, the yield for this configuration is then simply:

$\gamma_{\mathrm{B}}(2)=\mathrm{q} \times \frac{\mathrm{I}_{\mathrm{a}}-\mathrm{I}_{\mathrm{a} 0}}{\mathrm{I}_{2}}$

This configuration was only to be used with angles $\theta<70^{\circ}$ for the proton data presented in the next section. A careful comparison of earlier data obtained for all angles (also for oxygen and gold) showed a small but consistent difference of about $6 \%$ between results obtained with the two configurations consistent with a $6 \%$ of the electrons from the target being lost and not collected by the anode. Thus a $6 \%$ correction was applied to the five proton data points that had to be measured with the second configuration. The only other correction was a $\sim 8 \%$ upward adjustment of the three $0^{\circ}$ yields required by unavoidable electron losses through the apertures through which the beam enters for this angle (see fig. 1). This electron loss was measured at $20^{\circ}$ for each case by comparing results obtained by covering and uncovering the $0^{\circ}$ apertures. These losses for $0^{\circ}$ and for $20^{\circ}$ are expected to be identical for all practical purposes.

For each configuration, signal as a function of bias was measured and in both cases saturation was observed beyond 50 or 60 Volts. The data shown in the next section were obtained with bias voltages of 200 Volts.

An optical telescope was used to align the slits and the plate assembly. This telescope was also used to zero the angle encoder with a precision of about $0.1^{\circ}$. To obtain a more accurate adjustment the ratios of beam currents in FC2 and FC1 were plotted, and the zero-intercept of a straight line drawn through these data determines the $90^{\circ}$ angle with a precision of about $\pm 0.02^{\circ}$. An example of such a plot is shown in fig. 2

\section{Experimental Results}

The target material studied with these measurements was 304 stainless steel. The density is $8.02 \mathrm{~g} / \mathrm{cm}^{3}$ [19]. The composition this material can vary slightly around typical values [19]. The values adopted for the interpretation of the data are listed in Table 2. For $\mathrm{Cr}$ the specified concentration is $18-20 \%$, and $19 \%$ was adopted. For $\mathrm{Ni}$, $10 \%$ was adopted since the specified concentration is 8$12 \%$. For $\mathrm{Mn}$ and $\mathrm{Si}$ we adopted half the maximum values of $2 \%$ and $1 \%$ respectively. $\mathrm{C}, \mathrm{P}$ and $\mathrm{S}$ all have

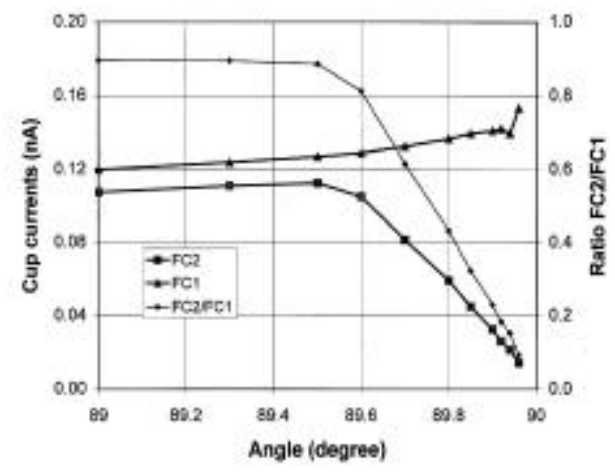

Figure 2. Example of the plots of Faraday cup currents and their ratio as a function of angles close to $90^{\circ}$ utilized to determine the zero for the angle encoder.

Table 2. Composition of 304 SS [19]

\begin{tabular}{||l||c||}
\hline Element & $\begin{array}{c}\text { Composition of \#304 SS } \\
\text { (\% in weight) }\end{array}$ \\
\hline \hline $\mathrm{Fe}$ & $69.5 \pm 4.5$ \\
\hline $\mathrm{Cr}$ & $19.0 \pm 2.0$ \\
\hline $\mathrm{Ni}$ & $10.0 \pm 1.0$ \\
\hline $\mathrm{Mn}$ & $1.0 \pm 1.0$ \\
\hline $\mathrm{Si}$ & $0.5 \pm 0.5$ \\
\hline
\end{tabular}


concentrations of less than $0.1 \%$, and they were neglected. These small uncertainties in the composition will have negligible impact on the interpretation of the data.

The ion beams used for these experiments are listed in Table 3, together with some of the quantities characteristic of their slowing down and their angular scattering in stainless steel.

Table 3. Ion beams used and their interaction with stainless steel

\begin{tabular}{|c|c|c|c|}
\hline Ion & $\begin{array}{c}\text { Proton } \\
\left({ }^{1} \mathrm{H}\right) \\
\end{array}$ & $\begin{array}{c}\text { Oxygen } \\
\left({ }^{16} \mathrm{O}\right)\end{array}$ & $\begin{array}{c}\text { Gold } \\
\left({ }^{197} \mathrm{Au}\right)\end{array}$ \\
\hline Energy $(\mathrm{MeV})$ & 28 & 126 & 182 \\
\hline Energy (MeV/amu) & 28 & 7.9 & 0.92 \\
\hline Charge state & $1+$ & $8+$ & $31+$ \\
\hline Electronic stopping power, $\mathrm{S}_{\mathrm{E}}\left(\mathrm{MeV} \mathrm{cm}^{2} / \mathrm{mg}\right)$ & 0.01342 & 2.093 & 53.81 \\
\hline Elastic stopping power, $\mathrm{S}_{\mathrm{N}}(\mathrm{MeV} \mathrm{cm} / \mathrm{mg})$ & $5.8 \times 10^{-6}$ & 0.00117 & 0.57 \\
\hline Linear energy transfer, LET $\left(\mathrm{MeV} \mathrm{cm}^{2} / \mathrm{mg}\right)$ & 0.01342 & 2.094 & 54.38 \\
\hline $\mathrm{S}_{\mathrm{N}} / \mathrm{LET}(\%)$ & 0.043 & 0.056 & 1.05 \\
\hline Range $(\mu \mathrm{m})$ & 1490 & 51.5 & 7.73 \\
\hline Mean square scattering angle $\mathrm{e}^{\dagger}$ for one micron $(\% / \mu \mathrm{m})$ & 0.22 & 0.54 & 0.98 \\
\hline
\end{tabular}

$\dagger$ This is the calculated mean square scattering angle [20] after $1 \mu \mathrm{m}$ of material for the trajectory projected onto a plane containing the incoming trajectory. For small scattering angles their mean square values will scale approximately as the square root of the thickness.

The stainless steel electron yields as a function of incidence angle obtained in these experiments for the three ion beams listed above are tabulated in Table 4. The data were obtained as described in section II except that, for the flat plate, average yield values from the "up" and "down" positions are used. These yields should in principle be identical. The use of these averages reduces random errors due, e.g., to beam fluctuations and, to first order, eliminates a possible systematic error due to small plate rotations coupled to the vertical displacements.

Least square fits were performed for the flat plate data between $0^{\circ}$ and $89^{\circ}$ to obtain estimates of the parameters in eq. 2 in section 1. Figure 3 shows double logarithmic plots of the yields as functions of the cosine of the angles. Deviations from the behavior described by eq. 2 are seen as departures from straight lines.

The fits are quite good for most of the angular range, and the deviations are discussed in the next section. The parameters resulting from this fitting procedure are listed in Table 5. The tabulated uncertainties are the purely statistical standard deviations resulting from the least square fitting procedure.
Table 4. Secondary electron yields (electrons per ion) measured for proton, oxygen and gold beams incident on flat and serrated stainless steel plates for different angles of incidence $\theta$. Normal incidence corresponds to $\theta=0^{\circ}$.

\begin{tabular}{||r||r|r||r|r||r|r||}
\hline \multicolumn{1}{||c|}{$\begin{array}{c}\theta \\
\text { (degree) }\end{array}$} & \multicolumn{1}{|c||}{ 28 MeV protons } & 126 MeV Oxygen & \multicolumn{2}{|c||}{ 182 MeV Gold } \\
\cline { 2 - 7 } & Flat & Serrated & Flat & Serrated & Flat & Serrated \\
\hline \hline 0.00 & 0.14 & 0.21 & 17 & 24 & 209 & 351 \\
\hline 20.00 & 0.14 & 0.37 & 18 & 36 & 223 & 578 \\
\hline 40.00 & 0.18 & 0.66 & 22 & 46 & 275 & 537 \\
\hline 50.00 & 0.23 & 0.40 & 27 & 18 & 325 & 251 \\
\hline 60.00 & 0.32 & 0.40 & 33 & 17 & 410 & 255 \\
\hline 70.00 & 0.47 & 0.43 & 49 & 19 & 598 & 265 \\
\hline 75.00 & 0.65 & 0.53 & 62 & 20 & 751 & 270 \\
\hline 80.00 & 0.96 & 0.92 & 93 & 21 & 1110 & 283 \\
\hline 82.00 & 1.23 & 1.02 & 117 & 23 & 1339 & 279 \\
\hline 84.00 & 1.73 & 1.20 & 150 & 25 & 1758 & 274 \\
\hline 85.00 & 2.18 & 1.23 & 174 & 25 & 2118 & 303 \\
\hline 86.00 & 2.92 & 1.36 & 218 & 25 & 2645 & 306 \\
\hline 86.50 & 3.52 & 1.40 & 247 & 27 & 2965 & 309 \\
\hline 87.00 & 4.18 & 1.59 & 282 & 27 & 3497 & 297 \\
\hline 87.50 & 5.24 & 1.68 & 341 & 30 & 4282 & 363 \\
\hline 88.00 & 6.86 & 1.84 & 422 & 30 & 5386 & 330 \\
\hline 88.50 & 8.93 & 1.91 & 591 & 32 & 7269 & 337 \\
\hline 89.00 & 13.68 & 1.96 & 967 & 36 & 10638 & 356 \\
\hline 89.30 & 19.54 & 2.02 & 1460 & 42 & 13457 & 396 \\
\hline 89.50 & 26.34 & 2.10 & 2210 & 47 & 16862 & 393 \\
\hline 89.60 & 32.41 & 2.17 & 2696 & 51 & 19114 & 392 \\
\hline 89.70 & 42.16 & & 3387 & & 23130 & \\
\hline 89.80 & 62.12 & & 4479 & & 26558 & \\
\hline 89.85 & 79.71 & & 5537 & & 30755 & \\
\hline 89.90 & 99.59 & & 6112 & & 32414 & \\
\hline 89.92 & 102.43 & & 6461 & & 32757 & \\
\hline 89.94 & 113.13 & & & & 30539 & \\
\hline 89.96 & 147.81 & & & & 28046 & \\
\hline \hline & & & & & & \\
\hline
\end{tabular}




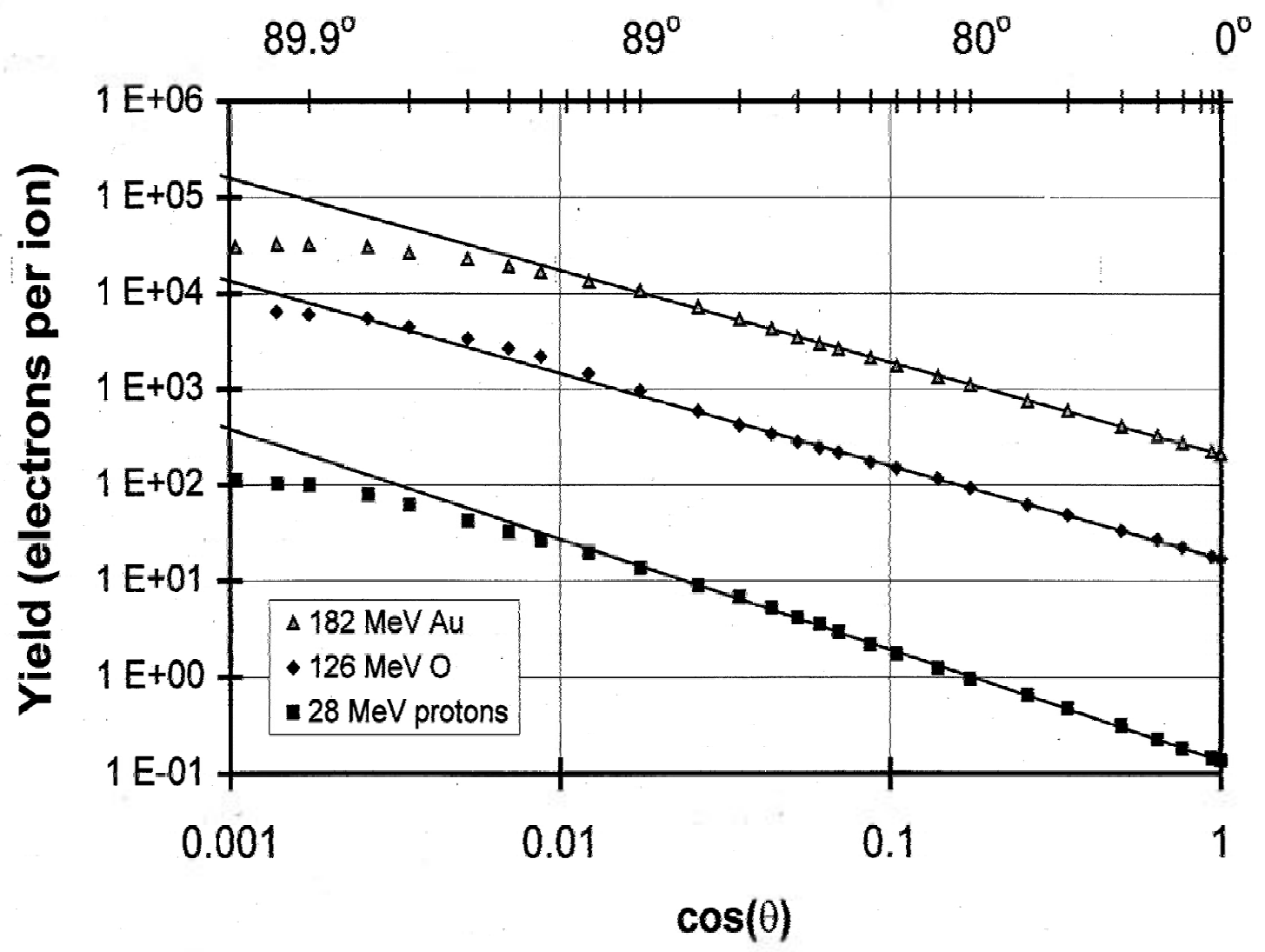

Figure 3. Doubly logarithmic plot of the flat plate yields listed in Table 4, vs. $\cos (\theta)$. The straight lines are results of least square fits of the data between $0^{\circ}$ and $89^{\circ}$ to the functional form given in eq. 2 with the resulting parameters listed in Table 5 .

Table 5. Parameters resulting from least square fitting of the data (see text).

\begin{tabular}{||c||c|c|c|c||}
\hline \hline \multicolumn{1}{|c||}{ Ion Beam } & $\gamma_{\mathrm{B}}\left(0^{\circ}\right)$ & $\mathrm{f}$ & $\begin{array}{c}\Lambda_{\mathrm{B}} \\
\left(\mathrm{mg} /\left(\mathrm{MeV} \mathrm{cm}^{2}\right)\right.\end{array}$ & $\mathrm{C}_{\mathrm{B}}$ \\
\hline \hline 28 MeV Protons & $0.135 \pm 0.003$ & $1.152 \pm 0.008$ & 10.06 & 1.0 \\
\hline $126 \mathrm{MeV}$ Oxygen & $16.96 \pm 0.30$ & $0.969 \pm 0.008$ & 8.10 & 0.805 \\
\hline $182 \mathrm{MeV}$ Gold & $208.1 \pm 2.5$ & $0.962 \pm 0.005$ & 3.87 & 0.385 \\
\hline
\end{tabular}

Here the parameters $\Lambda_{\mathrm{B}}$, as defined before, are the ratios $\gamma_{\mathrm{B}}\left(0^{\circ}\right) / \mathrm{S}_{\mathrm{E}}$ where $\mathrm{S}_{\mathrm{E}}$ are the electronic stopping powers listed in Table 3. The coefficients $\mathrm{C}_{\mathrm{B}}$ are the parameters $\Lambda_{\mathrm{B}}$ normalized to 1.0 for protons. They are thus a measure of the so called electron yield "deficit" with respect to the simplest predictions based on the proton results (see section 1).

Figures 4,5 and 6 show the data for the individual ion species, and compare in each case the flat plate with the serrated plate results. These plots are linear in the angle of incidence. The lines joining the points for the serrated plate data are for guiding the eye, and the lines for the flat plate are calculated with eq. 2 using the parameters listed in Table 5. 


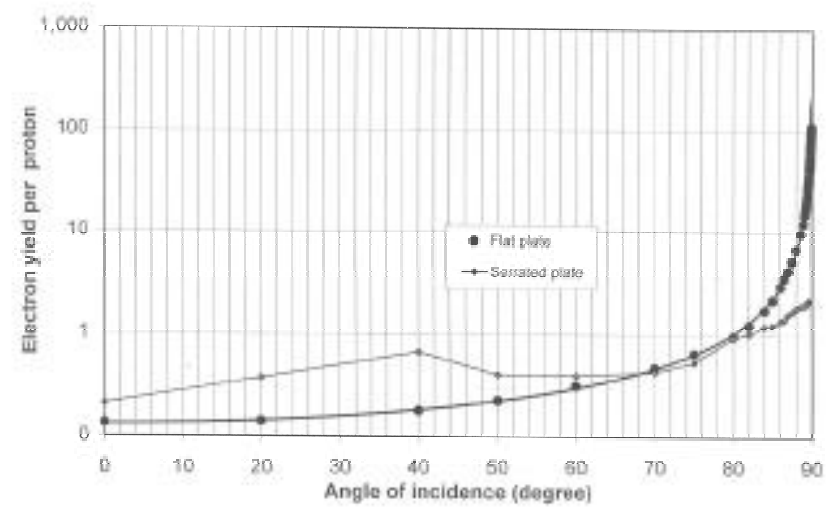

a

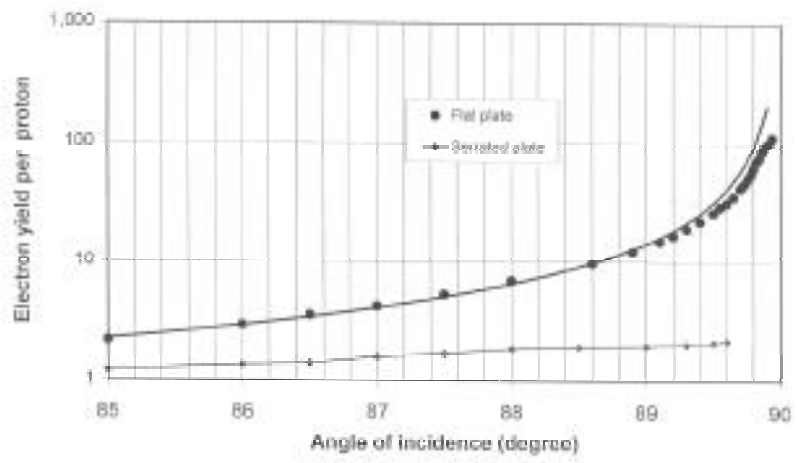

Figure 4.

a) Secondary electron yields vs. angle of incidence for 28 $\mathrm{MeV}$ protons striking a flat and a serrated stainless steel surface.

b) Expanded view of the last $5^{\circ}$.

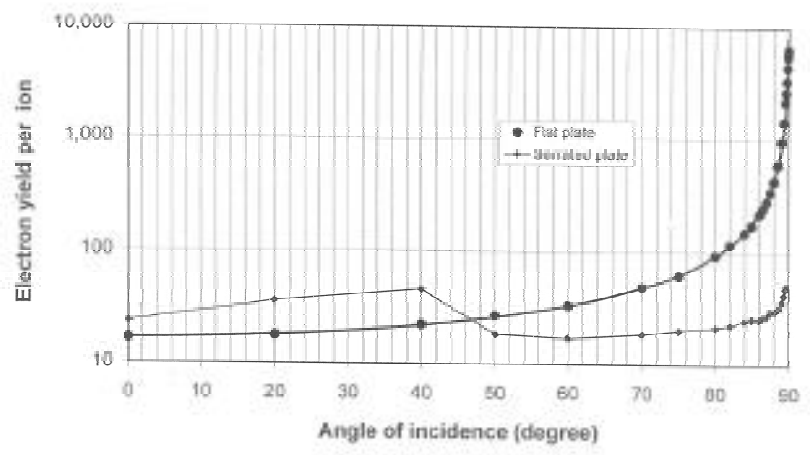

Figure 5.

a) Secondary electron yields vs. angle of incidence for 126 $\mathrm{MeV} 8+$ oxygen ions striking a flat and a serrated stainless steel surface.

b) Expanded view of the last $5^{\circ}$.

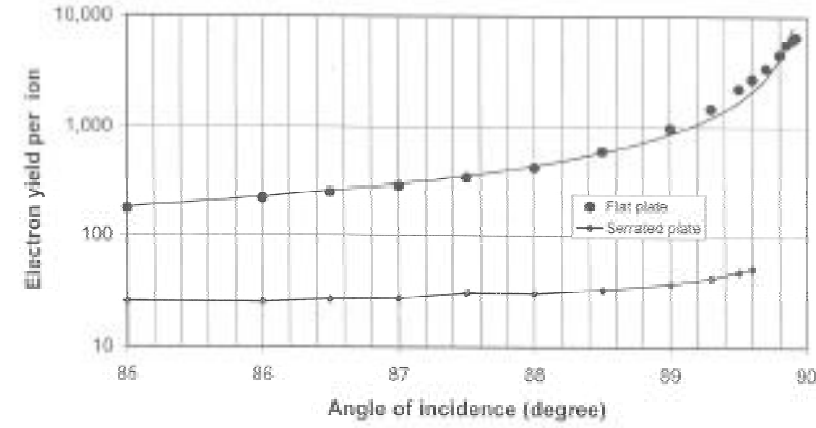

b 


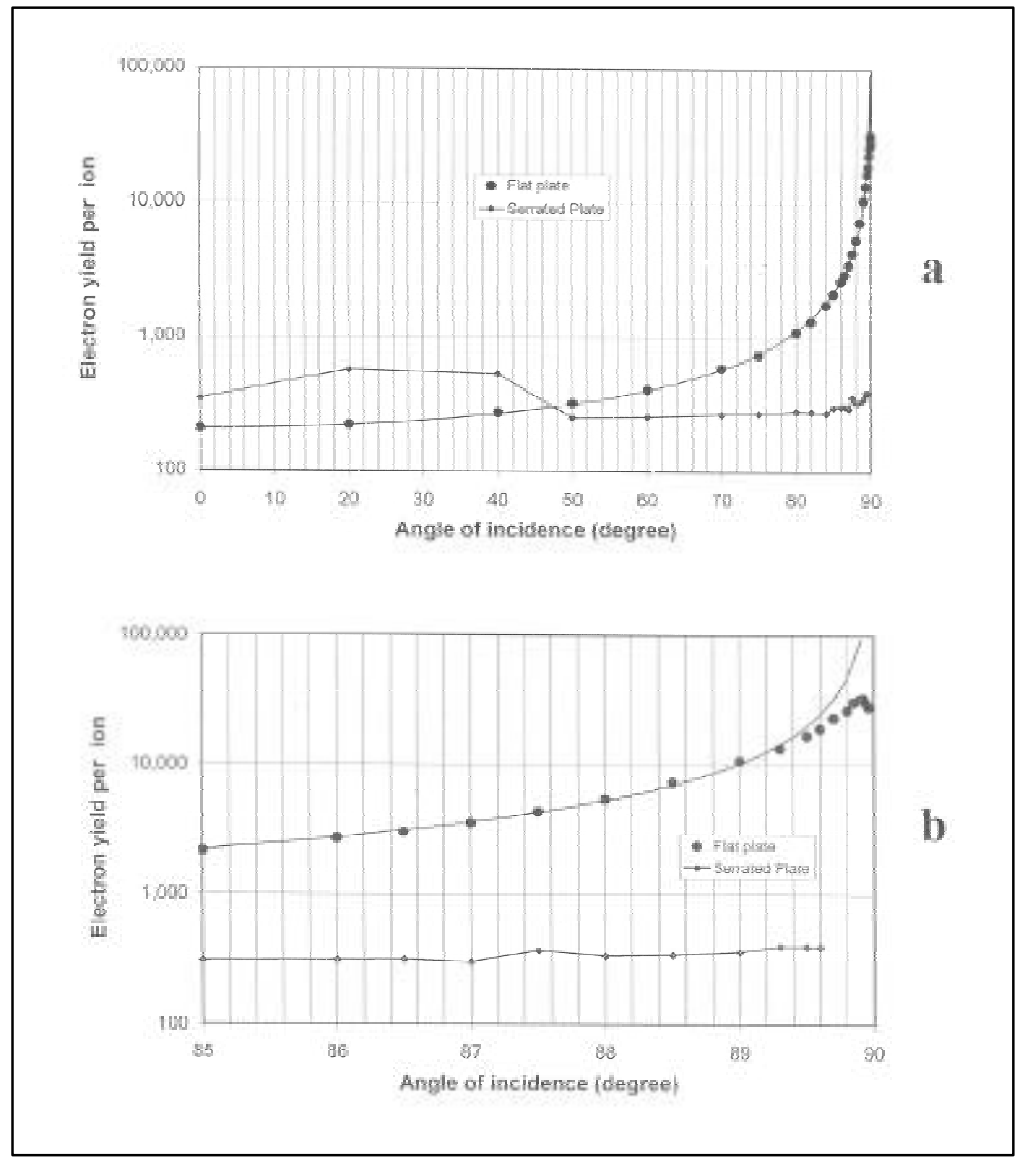

Figure 6.

a) Secondary electron yields vs. angle of incidence for 182 MeV 31+ gold ions striking a flat and a serrated stainless steel surface.

b) Expanded view of the last $5^{\circ}$.

\section{Discussion of the flat plate data and comparison with other results}

First we shall compare our proton results with the normal incidence yields obtained by others for various materials. Table 6 lists $\Lambda_{\mathrm{B}}$ values together with the corresponding beam energies or energy ranges. Far from a comprehensive review this is only a comparison of some of the more relevant data.

Table 6. Comparison of some of the electron yield data for normal incidence of energetic protons on various materials

\begin{tabular}{|c|c|c|c|}
\hline Target material & $\begin{array}{l}\text { Energy } \\
(\mathrm{MeV})\end{array}$ & $\begin{array}{l}\Lambda_{\mathrm{B}}=\gamma_{\mathrm{B}} /(\mathrm{dE} / \mathrm{dx})_{\mathrm{e}} \\
\mathrm{mg} /\left(\mathrm{MeV} \mathrm{cm}^{2}\right)\end{array}$ & References \\
\hline $\mathrm{C}$ & 0.02 to 7.5 & 5.0 & Ref. [13] \\
\hline $\mathrm{Al}_{2} \mathrm{O}_{3}$ & to 67 & $9.4 \pm 0.6$ & Calculated from data in ref. [14] \\
\hline $\mathrm{Au}$ & to 18 & $22 \pm 3$ & Calculated from data in ref. [21] \\
\hline $\mathrm{Al}$ & \multirow{4}{*}{4 to 12} & 3.6 & \multirow{4}{*}{$\begin{array}{l}\text { Calculated from data in ref. [22] } \\
\text { and [23] }\end{array}$} \\
\hline $\mathrm{Cu}$ & & 7.3 & \\
\hline $\mathrm{Ag}$ & & 13.1 & \\
\hline $\mathrm{Au}$ & & 18.9 & \\
\hline 304 stainless steel & 28 & $10.1 \pm 0.3$ & Present work \\
\hline
\end{tabular}

One sees, as mentioned before, that the values of $\Lambda_{\mathrm{B}}$ have proven to be fairly constant for each material in experiments covering wide energy ranges. There apparently are no proton data beyond $67 \mathrm{MeV}$. However, if one assumes that $\Lambda_{\mathrm{B}}$ will remain approximately constant up to $1 \mathrm{GeV}$ then, using our results, one can 
estimate the electron yield for SS at that energy. The value of the electronic stopping power $(\mathrm{dE} / \mathrm{dx})_{\mathrm{e}}$ is calculated [20] to be $1.61 \times 10^{-3} \mathrm{MeV} \mathrm{cm}^{2} / \mathrm{mg}$. Then assuming that the value of $\Lambda_{\mathrm{B}}$ remains constant at $10.1 \mathrm{mg} /(\mathrm{MeV} \mathrm{cm})^{2}$, we get an estimate of 0.016 electrons per $1 \mathrm{GeV}$ proton for the yield at normal incidence on a 304 stainless steel surface.

Our oxygen and gold beam results are in line with the well known fact [2] that for heavier and heavier ions secondary electron yields per unit LET deviate increasingly from the proton values. In other words, as can be seen from Table 5, $1>\mathrm{C}_{\mathrm{B}}(\mathrm{O})>\mathrm{C}_{\mathrm{B}}(\mathrm{Au})$ which means that the heavier ions are less efficient in converting into secondary electrons some of the energy deposited in the surface layer. More detailed comparisons with other results is difficult since data are still rather sparse, in particular for the higher beam energies, and the parameter space is very large considering the possible combinations of ion species, beam energies and target materials.

Of the several possible explanations for the electron "deficit" for heavier ions, one of the most frequently mentioned is based on the very thin escape zone and on the fact that charge state equilibrium can not be reached within that layer. This pre-equilibrium near-surface stopping power concept [16] can not explain our heavy ion results, in particular not the ones for gold. In this case the incoming charge state of $31+$ is much higher than the "effective" charge state $\sim 19+$ deduced from the bulk stopping power data. So, if stopping power and consequently electron emission scale as the square of the charge, one should naively expect an enhancement of $\sim 2.7$ rather than the observed deficit $\left(C_{B}=0.38\right.$, Table 5). Clearly, at least for this ion-target combination, other factors must be responsible for the deficit.

We finally turn to the results for the incidence angle dependence of the yield, the study of which was the main thrust of the present work. Very few relevant references were found and the one covering the widest angular range [17] shows results ranging from $0^{\circ}$ to only $85^{\circ}$ obtained with various $40 \mathrm{keV}$ ions on a copper target. Another experiment [24], using $100 \mathrm{MeV}$ Si ions on various metallic targets and on silicon, was limited to an angular range from $10^{\circ}$ to $70^{\circ}$.

As is seen most clearly from fig. 3, we find excellent agreement with the angular dependence described by eq. 2 over an angular range $89^{\circ}>2>0^{\circ}$ which, compared to previous experiments [17,24], extends to angles much closer $90^{\circ}$. To see if the deviations observed for angles starting at $\sim 89^{\circ}$ could be due to the multiple scattering or energy loss mechanisms mentioned in section 1 , we will first try to estimate these effects at $2=89^{\circ}$ :

We must first calculate the length of that part of the trajectory which is within the surface layer from which electrons may escape. The thickness of this layer was estimated to be roughly $30 \AA$ for a carbon target [8]. Since it is the electrons in the material that are responsible for stopping the escaping electrons, it is reasonable to reduce this escape depth from carbon to SS by the ratio of their electron densities. This ratio is roughly equal to 0.3 , which leads to an escape depth for SS of about $9 \AA$. The corresponding path length within the escape zone is thus $9 \AA / \cos \left(89^{\circ}\right)=516 \AA \approx 0.05 \mu \mathrm{m}$. This path length is so small compared to even the smallest ion range $(7.7 \mu \mathrm{m}$ for the gold beam, see Table 3$)$ that changes in LET during ion penetration can not account for the observed effect. The calculated multiple scattering angles for $1 \mu \mathrm{m}$ (see Table 3) are also so small that angular changes of individual trajectories are unlikely to account for an appreciable part of this effect in the vicinity of $89^{\circ}$.

We turn now to the maximum in the yield curve which we observed for the gold data around $89.9^{\circ}$ (see fig. 6b). Svensson, et.al. [17] using $40 \mathrm{keV}$ protons, had observed maxima in their yield vs. angle curves at angles between $78^{\circ}$ and $82^{\circ}$ for the various target materials. They suggest that these maxima could be correlated with the angles at which sputter yield maxima occur. Repeating the calculation of these maximum sputter yield angles for the ions used in the present experiments we should expect maximum secondary electron yields at $88.5^{\circ}$ for the gold beam, at $88.9^{\circ}$ for the oxygen beam and $89.0^{\circ}$ for the protons. It is clear from Table 4 and figs. 4,5 and 6 that in our data the only maximum observed is at $\sim 89.9^{\circ}$ for the gold beam, and that there are no maxima at least up to $89.92^{\circ}$ for oxygen and $89.96^{\circ}$ for protons. Either the suggested correlation, for some reason, stops working at the higher energies, or the effect observed by Svensson, et.al. was due to other physical or instrumental effects.

We will now see if the maximum we observed at $89.9^{\circ}$ for the gold beam could be due to multiple scattering. For a particle incident at $89.9^{\circ}$ the path length within the $\sim 9 \AA$ deep surface layer which 
corresponds to the electron escape zone is $9 \AA / \cos \left(89.9^{\circ}\right)=0.52 \mu \mathrm{m}$ if multiple scattering can be neglected. If multiple scattering is not negligible, then some of the ions will be driven faster into the bulk of the material, some will be driven out and "reflected" and a very few may stay longer within the escape zone. The overall effect will be a reduction of the electron yield. To evaluate the possible effects of multiple scattering on the electron yields around $89.9^{\circ}$ we calculate for each of the ion beams the mean square scattering angle after the first half of the escape zone is penetrated, i.e. after $0.26 \mu \mathrm{m}$ :

\begin{tabular}{||l||c|}
\hline Ion beam & Scattering angle \\
\hline \hline $28 \mathrm{MeV}$ protons & $0.047^{\circ}$ \\
$126 \mathrm{MeV}$ oxygen & $0.086^{\circ}$ \\
$182 \mathrm{MeV}$ gold & $0.556^{\circ}$ \\
\hline
\end{tabular}

Table 7. Calculated mean square projected scattering angles [20] of the three ions used in the experiment after penetrating half of the electron escape-depth at $89.9^{\circ}$ incidence.

Without attempting a detailed quantitative argument we see that only for the gold beam is the multiple scattering very significant at this angle. For oxygen the ions must travel through half escape zone before the mean square angle becomes comparable to $90^{\circ}-\theta$ and therefore the effect will be small, and it will be even smaller for protons. We therefore conclude that multiple scattering may be a plausible explanation for having observed a maximum only for the gold beam, even though we of course realize that these arguments get more complicated if surface topography is considered.

It should finally be mentioned that a number of recent publications (see e.g. $[25,26]$ ) present predictions and results for grazing collisions but only low energy $(<1 \mathrm{MeV})$ experimental data were available and total electron yields are not always addressed. Hopefully our results will stimulate calculations for higher energies, which may then serve to obtain better extrapolations to, e.g., $1 \mathrm{GeV}$ protons.

\section{Discussion of the serrated plate data and prospects for higher energies}

The idea of reducing grazing incidence secondary electron yields by replacing flat electrodes by serrated surfaces is based on the following facts:

a) For the accelerator applications of interest, most of the ions causing the secondary electrons (e.g. halo particles), far from being istropically distributed in space, are instead highly collimated within a small solid angle centered on the main beam direction.

b) Most of the surface area defining the individual teeth of such a serrated plate is inclined with respect to the incoming ion trajectories by large angles ( $\pm 45^{\circ}$ for our experiment).

c) The backward secondary electron yield at the impact point with such an inclined surface is much smaller than at grazing incidence with a flat plate. But of course electrons will also be generated at the exit points for ions penetrating the teeth and at subsequent impact points, etc.

To see to what extent our data confirm such yield reductions we compare in Table 8 grazing incidence on both type of surfaces with $45^{\circ}$ flat plate results. For grazing incidence we selected $89.6^{\circ}$ from Table 4 , which is the largest angle for which we have useful serrated plate data. The $45^{\circ}$ yields shown in Table 8 were obtained by linear interpolation between the $40^{\circ}$ and $50^{\circ}$ values in Table 4 .

\begin{tabular}{||l||l|c|c||}
\hline \hline Ion Beam & $\begin{array}{l}\text { Flat plate } \\
\text { yield at } 89.6^{\circ}\end{array}$ & $\begin{array}{l}\text { Serrated plate } \\
\text { yield at } 89.6^{\circ}\end{array}$ & $\begin{array}{l}\text { Flat plate } \\
\text { yield at } 45^{\circ}\end{array}$ \\
\hline \hline $28 \mathrm{MeV}$ protons & 32.4 & 2.17 & 0.21 \\
\hline $126 \mathrm{MeV}$ oxygen & 2696 & 50.6 & 24.7 \\
\hline $182 \mathrm{MeV}$ gold & 19114 & 392 & 300 \\
\hline
\end{tabular}

Table 8. Comparison of near grazing incidence secondary electron yields for the flat and serrated plates with $45^{\circ}$ incidence yields on the flat plate. 
We see that, at $89.6^{\circ}$, the serrated plate yields are indeed much smaller than the flat plate yields, but not as small (especially for protons) than the flat plate yields at $45^{\circ}$. It is easy (neglecting multiple scattering) to calculate from the ranges listed in Table 3 , and from the geometry indicated in fig. $7 \mathrm{a}$ with $\mathrm{s}=2 \mathrm{~h}=12.7$ $\mathrm{mm}$, that $28 \mathrm{MeV}$ protons incident at an angle $\theta=89.6^{\circ}$ will traverse three or four teeth before stopping. This would correspond to 7 or 9 traversals of solid-vacuum boundaries. Considering that exit yields are generally somewhat larger than entrance yields (Meckbach factors of $~ 1.3$ are common), and the fact that LET values increase as the protons slow down, it is not surprising to find the serrated plate yield $\sim 10$ times larger than the flat plate yield at $45^{\circ}$. In fact an even larger yield would be expected if it were not for multiple scattering causing considerable deflection after a few $100 \mu \mathrm{m}$ of penetration (see Table 3). No attempt was made at performing a Monte Carlo-type calculation to take this effect into account. Finally it should be mentioned that the surface polish of the serrations is not quite as good as for the flat plate, and this fact will also affect the comparison.

Similar considerations applied to the oxygen and gold beams show that at $89.6^{\circ}$ only a fraction of these ions ( $29 \%$ for oxygen and $\sim 5 \%$ for gold) will manage to traverse a single tooth (3 interface traversals) while the rest are stopped after penetrating the first surface. Also, the geometry that would need to be considered is no longer as simple as indicated in fig. 7a because now the ranges are comparable to the radius of curvature characteristic of the upper edges of the serration teeth. Thus, grazing (high yield) collisions close to these edges become more significant. In view of these considerations, and without attempting detailed estimates, it seems that the values shown in Table 8 are also very reasonable for the oxygen and gold beams.

Finally we will attempt to provide estimates of serrated surface electron yields for the case of $1 \mathrm{GeV}$ protons, to see if this is a promising approach for the SNS collimator design [27]. From what we have learned, it should indeed be easier to perform valid calculations at this energy since multiple scattering and energy loss, will be less important, and can at first be neglected. For simplicity we will also assume a $1 / \cos (\theta)$ angular yield dependence, which for the present purpose is close enough to behavior observed at lower energies (see Table 5).
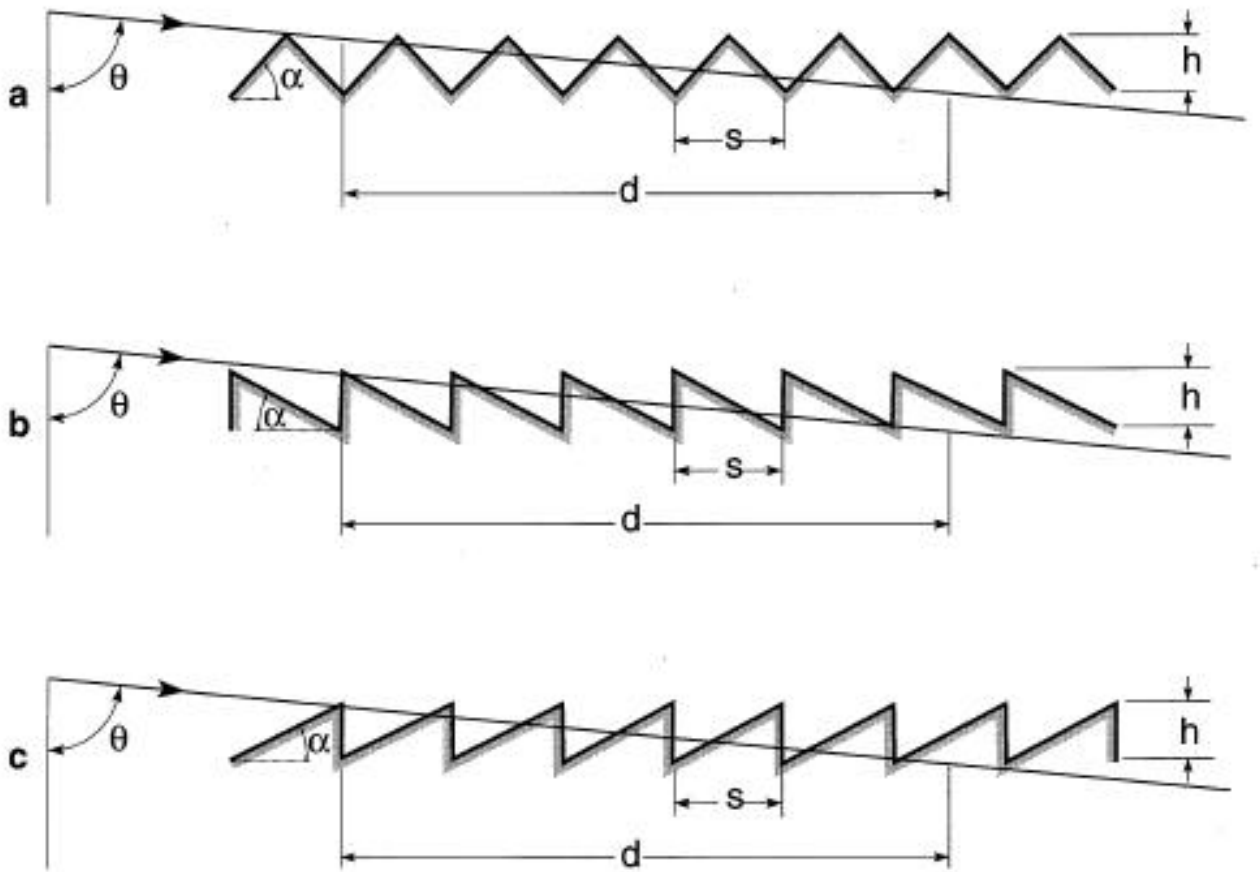

Figure 7. Schematic cross sections of serrated plate surfaces used as examples for yield estimates (see text). Fig. $7 \mathrm{a}$ with $\mathrm{s}=2 \mathrm{~h}=12.7 \mathrm{~mm}$ corresponds to the serrated plate used in the present experiments. 
We performed calculations for the three serration-teeth geometries illustrated in figs $7 \mathrm{a}, 7 \mathrm{~b}$, and $7 \mathrm{c}$ to evaluate possible effects of different shapes on secondary emission performance. Considering first the case illustrated in fig. 7a, and calling $\pm \alpha$ the inclination of the serration surfaces, $s$ the distance between teeth, $h$ the depth of the teeth and $d$ the longitudinal distance for a particle coming in at an incident angle $\theta$ to penetrate through a depth $\mathrm{h}$, we can write:

$\mathrm{d}=\mathrm{h} \operatorname{tg}(\theta)$

$s=\frac{2 h}{\operatorname{tg}(\alpha)}$

And calling $\mathrm{N}$ the number of teeth traversed we get

$\mathrm{N}=\frac{\mathrm{d}}{\mathrm{S}}=\frac{\operatorname{tg}(\alpha) \operatorname{tg}(\theta)}{2}$

Which for large $\mathrm{N}$ is also approximately equal to the number of incoming and the number outgoing surface traversals of the particle before it gets buried in the bulk of the electrode. Calling $\gamma_{\mathrm{TB}}$ and $\gamma_{\mathrm{TF}}$ the total backward and forward (incoming and outgoing) electron yields for the $\mathrm{N}$ transitions, we get with the above mentioned assumptions:

$\gamma_{\mathrm{TB}}=\mathrm{N} \frac{\gamma_{0}}{\cos (\theta-\alpha)}$

$\gamma_{\mathrm{TF}}=-\mathrm{N} \frac{\mathrm{M} \gamma_{0}}{\cos (\theta+\alpha)}$

where $\gamma_{0}$ is the normal incidence backward yield, and $\mathrm{M}$ is the Meckbach factor, i.e. the enhancement of forward vs. backward yield.

From 7), 8) and 9) we get the total yield $\gamma_{\mathrm{T}}=\gamma_{\mathrm{TB}}+\gamma_{\mathrm{TF}}$ :

$\left.\gamma_{\mathrm{T}}=\gamma_{0} \frac{\operatorname{tg}(\alpha) \operatorname{tg}(\theta)}{2}\left(\frac{1}{\cos (\theta-\alpha)}-\frac{M}{\cos (\theta+\alpha)}\right) 10\right)$

For angles close to grazing incidence, i.e. $\theta \rightarrow 90^{\circ}$ we get:

$$
\begin{aligned}
& \gamma_{\mathrm{T}} \rightarrow \gamma_{0} \frac{\operatorname{tg}(\alpha)}{2 \cos (\theta)} \frac{1+\mathrm{M}}{\sin (\alpha)} \\
& \gamma_{\mathrm{T}} \approx \gamma_{0} \frac{1+\mathrm{M}}{2 \cos (\alpha) \cos (\theta)}
\end{aligned}
$$

Comparing to the yield $\gamma_{\text {flat }}$ from a flat plate at the same incident angle we get:

$\frac{\gamma_{\mathrm{T}}}{\gamma_{\mathrm{flat}}}=\frac{1+\mathrm{M}}{2 \cos (\alpha)}$

Since $M>1$ (typically 1.3 or 1.5 ) and $\cos (\alpha)<1$ we see that this ratio is always larger than 1 and therefore we would get more secondary electrons from the serrated plate. Note that this result is independent of the absolute yield for normal incidence and depends only on the $1 / \cos (\theta)$ assumption for the angular dependence and on the approximations of negligible change in $(\mathrm{dE} / \mathrm{dx})_{\mathrm{e}}$ and negligible scattering while penetrating the serration depth.

The same approach was followed for the geometry shown in fig. $7 \mathrm{~b}$ where the entrance face of the serration teeth is perpendicular to the overall electrode surface. In this case we get: 


$$
\frac{\gamma_{\mathrm{T}}}{\gamma_{\mathrm{flat}}}=\operatorname{tg}(\alpha)+\frac{\mathrm{M}}{\cos (\alpha)}
$$

We see that for small values of $\alpha$ the limit of this expression is M, which is larger than 1. Again, there is no advantage in using a serrated plate. By inverting the teeth (see fig. 7c) we get

$$
\frac{\gamma_{\mathrm{T}}}{\gamma_{\text {flat }}}=\mathrm{M} \operatorname{tg}(\alpha)+\frac{1}{\cos (\alpha)}
$$

This is better, since now the same yield as obtained from a flat plate can be approached for small angles $\alpha$. In a practical application the smallest allowable $\alpha$ is determined by the angular distribution of the incident particles since an abundance of grazing collision on the inclined teeth surfaces would defeat the purpose of a serrated surface. For e.g. $\alpha=10^{\circ}$ and $M=1.5$ we get $\gamma_{\mathrm{T}} / \gamma_{\text {flat }}=1.28$. The serrated plate performance is still slightly worse when compared to the flat plate as also indicated by the dotted lines in fig. 8 .

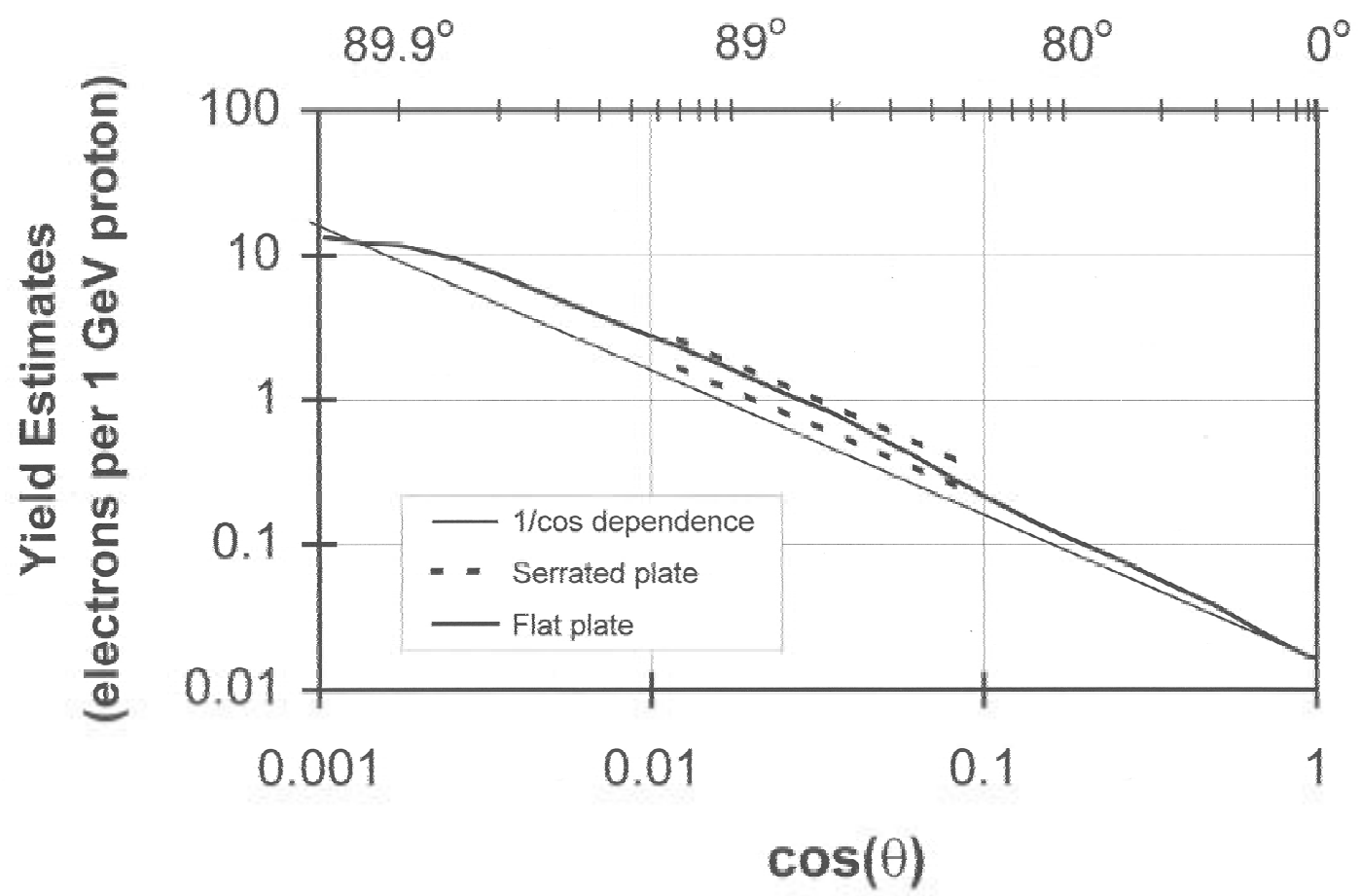

Figure 8. Estimated electron yields for $1 \mathrm{GeV}$ protons incident on smooth (solid lines) and serrated (dotted lines) SS surfaces. The curved line represents the present data scaled from $28 \mathrm{MeV}$ to $1 \mathrm{GeV}$ by the ratio of the respective stopping powers. The straight solid line shows a $\cos ^{-1}(\theta)$ angular dependence. The dotted lines correspond to a serrated plate such as depicted in fig. 7c with a tooth height $\mathrm{h}=5 \mathrm{~mm}$ and $\alpha=10^{\circ}$ (see text). The upper and lower dotted lines were obtained assuming $\cos ^{-1.152}(\theta)$ and $\cos ^{-1}(\theta)$ angular dependencies, respectively. The left termination of these dotted lines indicates the incidence angle at which the calculated [20] mean square multiple scattering angle will be equal to $90^{\circ}-\theta$ after only half the involved teeth have been penetrated. The actual yield at this angle will already be reduced compared to what is shown, and beyond this angle strong multiple scattering effects will limit any further yield increase.

What will change this situation is multiple scattering. The increasing amount of serrated plate teeth material traversed as $90^{\circ}$ incidence angles are approached is orders of magnitude larger than the thin escape zone material traversed in the flat plate for the same angles. So multiple scattering will interrupt the yield increase for the serrated plate much sooner than for the flat plate. To estimate at what incidence angle this will happen we select as example serrations such as the ones depicted in fig. $7 \mathrm{c}$ with $\mathrm{h}=5 \mathrm{~mm}$ and $\alpha=10^{\circ}$, and we calculate the mean square scattering angle after a distance $d / 2$, when half the teeth have been 
penetrated. For this example TRIM calculations [20] for $1 \mathrm{GeV}$ protons in $304 \mathrm{SS}$ indicate that this scattering angle becomes equal to the angle $90^{\circ}-\theta$ between the surface and the incoming trajectory when $\theta=89.3^{\circ}$. Thus, beyond $\sim 89.3^{\circ}$ we can expect that the straight line increase indicated by the dotted lines in fig. 8 will not continue. For this angle, $\mathrm{d} / 2 \approx 20 \mathrm{~cm}$ and the equivalent thickness of SS traversed at the $\mathrm{d} / 2$ point is $\sim 5 \mathrm{~cm}$ with an energy loss of $65 \mathrm{MeV}$. The range of $1 \mathrm{GeV}$ protons in stainless steel is $\sim 57 \mathrm{~cm}$. Preliminary results form Monte Carlo-type calculations [28] tend to confirm these estimates.

We conclude that replacing smooth by serrated electrodes can be effective in reducing the secondary electron emission. In the case of our application of collimators for $1 \mathrm{GeV}$ protons, the tradeoff will be a slight increase in the penumbra of partially degraded protons. The design of the surface and the degree of the achievable electron reduction will depend on the angular distribution of the incident protons. For example, if the protons are restricted to a $\sim 1^{\circ}$ angular range between $89^{\circ}$ and $90^{\circ}$ then the $5 \mathrm{~mm}$ serrations described above should be quite effective. For wider incident distributions or for even better electron suppression deeper serrations may be required. Compared to similar electron reduction schemes [29], where the multiple scattering and collimating functions are separated, the present solution is probably more effective and it does encroach less on the useful collimator aperture.

\section{Conclusions}

Angular accuracy unprecedented for this type of measurements, as well as a much longer target and a novel system for compensating for lost beam at grazing angles, allowed electron yield measurements for incidence angles much closer to $90^{\circ}$ than hitherto possible. Near $1 / \cos (\theta)$ behavior was observed between $0^{\circ}$ and $89^{\circ}$ and the deviations from this behavior were accurately determined as well as the normal incidence yields. For $28 \mathrm{MeV}$ protons the electron yield is closely approximated by $0.135 / \cos (\theta)^{1.152}$ for $0^{\circ}<\theta<89.5^{\circ}$

Rather large grazing incidence yields of up to $~ 33,000$ electrons per ion were measured for the $1 \mathrm{MeV} / \mathrm{amu}$ gold beam, as expected from previous observations at accelerator related systems[6]. These data are thus directly relevant for practical applications. Together with the proton and oxygen data they should also be useful in extending and verifying existing or new theoretical descriptions, thus enhancing the understanding of the underlying phenomena and allowing more reliable predictions and extrapolations which are important for future accelerator applications.

Based on the present proton data, a preliminary estimate was obtained of 0.016 electrons per $1 \mathrm{GeV}$ proton for the yield at normal incidence on stainless steel. This result is of interest for the collimators being designed for the SNS facility. Using serrated surfaces in these collimators may solve the problem if the $\sim 1 / \cos (\theta)$ angular dependence leads to excessive electron production for grazing collisions of halo particles.

The present experiments can of course be extended to other materials, and coatings that have been suggested $[30,31]$ for the reduction of electron produced secondary electrons will be investigated. Also further improvements are possible in angular accuracy and in the small angle limit for these measurements. This would be of particular interest to find maxima for the oxygen and proton data and to compare their positions with the maximum found here for the gold beam at $\sim 89.9^{\circ}$. A more precise goniometer would be required to move the plate assembly, and the surface of the target would need to be machined or ground and polished to even higher accuracy.

\section{Acknowledgements}

This work was performed under the auspice of the U. S. Department of Energy. We would like to thank W. W. Weng for his encouragement and support, M. Manni for the detailed design and for supervising the fabrication of the hardware, D. A. Graham and H. F. Abendroth for assistance with electronics and computers, C. W. Carlson for his help with the alignment, and the BNL Tandem crew for providing the ions and invaluable support. 


\section{References}

1. J. Devooght, J.C. Dehaes, A. Dubus, M. Cailler, J.P. Ganachaud, M. Rosler, and M. Brauer, in Particle Induced Electron Emission I, edited by G. Hohler and E. A. Niekish, Springer Tracts in Modern Physics Vol. 122 (Spronger-Verlag, Berlin, 1991)

2. D. Hasselkamp, H. Rothard, K. O. Groeneveld, J. Kemmler, P. Varga , and H. Winter, in Particle Induced Electron Emission II, edited by G. Hohler and E. A. Niekish, Springer Tracts in Modern Physics Vol. 123 (Spronger-Verlag, Berlin, 1991)

3. H. Rothard, Scanning Microsc. 9, 1 (1995)

4. Ionization of Solids by Heavy Particles, Vol. 306 of NATO Advanced Study Institute, Series B: Physics, edited by R. Baragiola (Plenum, New York, 1993)

5. W. T. Weng, et.al., Proceedings of Particle Accelerator Conference, pp. 970-972, 1997, Vancouver, Canada.

6. S.Y. Zhang Proceedings of Particle Accelerator Conference, pp. 3297-3299, 1999, New York, NY, USA.

7. Y. Chanut, J. Martin and R. Salin, Surface Science 106, 563 (1981)

8. M. Jung, H. Rothard, B. Gervais and J. P. Grandin, Phys. Rev. A54 No5, 4253 (1996)

9. H. Rothard, J. Schou, K. O. Groeneveld, Phys. Rec. A45 No 3, 1701 (1992)

10. H. Rothard, C. Caraby, A. Cassimi, B. Gervais, J. P. Grandin, P. Jardin and M. Jung, Phys. Rev. A51, No 4, 3066 (1995)

11. E. J. Sternglass, Phys. Rev. 108,1 (1957)

12. W.Meckbach, G. Braunstein and N. Arista, J. Phys. B8, L344 (1975)

13. H. Rothard, M. Jung, M. Caron, J. P. Grandin, B. Gervais, A. Billebaud, A. Clouvas, and R. Wunsch, Phys. Rev. A57, No 5, 3660 (1998)

14. C. M. Castaneda, L. McGarry, C. Cahill, T. Essert, Nucl. Inst. and Meth. in Phys. Res. B129, 199 (1997)

15. H. Rothard, K. Kroneberger, A. C. Clouvas, E. Veje, P. Lorenzen, N. Keller, J. Kemmler, W. Meckbach, and K. O. Groeneveld,Phys. Rev. A, 41 No 5, 2521 (1990)

16. H. Rothard, J. Schou, and K. O. Groeneveld, Phys. Rev. A45, No 3, 1701 (1992)

17. B. Svensson, G. Holmén, and A. Burén, Phys. Rev. B24, 3749 (1981)

18. T. Roser, Fourth European Particle Accelerator Conference, pp. 151-155, 1994, London

19. Handbook of Chemistry and Physics, CRC Press, Inc, $71^{\text {st }}$ Edition, 12-122 (1991)

20. Calculated using an updated version of the TRIM program. J.F. Ziegler and J. P. Biersack 'The Stopping Power and Ranges of Ions in Matter", New York: Pergamon, 1985.

21. J. E. Borovsky, D.J. McComas, and B. L. Barraclough, NIM B30, 191 (1988)

22. D. Hasselkamp, S. Hippler, A. Scharmann, and T. Schmehl, Annalen der Physik,7.Folge, Band 47, Heft 7, 555 (1990)

23. A. Koyama, T. Shikata, and H. Sakairi, Jap. J. Appl. Phys. 20, 65 (1981)

24. R.S. Chauhan, V. K. Mittal, T. K. Nandi, A. Mandal and D. K. Avasthi, Vacuum 48 No 12 (1997) 1031

25. M.L.Martiarena, E.A. Sánchez, O.Grizzi, and V.H. Ponce, Phys.Rev. A53, 895 (1996)

26. M. S. Gravielle, Phys. Rev. A58, 4622 (1998)

27. H. Ludewig, et. al. , Proceedings of Particle Accelerator Conference, pp. 548-550, 1999, New York, NY, USA.

28. V. Zajic, et. al. (to be published)

29. N.N. Catalan Lasheras, F. Ferioli, J.B. Jeanneret, R. Jung, and T. Trenkler, "Proton Collimation in TeV Colliders", Proceedings, Near Beam Physics Symposium, Fermilab, Sept. 1997, pp117-126. Also LHC Project Report 156, Geneva, October 1997.

30. O. Bruning, et. al. "Electron Cloud and Beam Scrubbing in the LHC", Proceedings of the 1999 Particle Accelerator Conference.

31. K. Kennedy, et al, "TiN coating of the PEP-II Low Energy Ring Al Arc Vacuum Chambers" Proceedings of the 1997 Particle Accelerator Conference 